INVESTIGACIONES

\title{
PROCESOS COGNITIVOS IMPLICADOS EN LA LECTURA DE LOS SORDOS*
}

\author{
Cognitive process implicated deaf reading
}

\author{
Valeria Herrera F. \\ Dirección de Postgrados, Universidad Metropolitana de Ciencias de la Educación, \\ Calle Dr. Luis Bisquert S. 2765, Nuñoa, Santiago. Chile. \\ Valeria.herrera@umce.cl / valeria2hf@yahoo.com
}

\section{Resumen}

El estudio del aprendizaje de la lectura en los estudiantes sordos ha transitado desde posturas centradas en el déficit, como la falta de audición, a posturas que intentan explicar el fenómeno considerando las características específicas de estos estudiantes. Esta última postura ha llevado a explorar con una mirada distinta los procesos cognitivos implicados en la lectura de los sordos. Si bien los procesos en que se sustenta el aprendizaje lector son los mismos independientemente del estatus auditivo del sujeto, las estrategias de aprendizaje y los códigos de acceso a la información escrita parecen ser diferentes.

Palabras clave: lectura, aprendizaje, sordera.

\begin{abstract}
Research on reading learning by deaf students has gone from a focus on their deficits such as lack of auditory capabilities to wider views attempting to explain the phenomenon based upon specific visual characteristics of the students. This wider view has lead to explore the cognitive processes involved in deaf reading from new perspectives, which suggests that although the basic processes underlying reading learning are the same for people, regardless their auditory status, both the learning strategies used and the codes of access to the written information seem to be different for deaf people.
\end{abstract}

Key words: reading, learning, deafness.

* $\quad$ Este trabajo se ha realizado en el marco del Proyecto FONDECYT N ${ }^{\circ} 11075018$. 


\section{INTRODUCCION}

Durante décadas se argumentó que el principal obstáculo en el aprendizaje lector de los estudiantes sordos era el desconocimiento del lenguaje oral. Más recientemente, los estudios han centrado su interés en los procesos cognitivos implicados en el aprendizaje de la lectura. En este sentido, algunos autores señalan que las dificultades en lectura de los sordos se deben a problemas en la aplicación de las Reglas de Correspondencia Grafema-Fonema (en adelante RCGF) y en la capacidad de almacenamiento de la Memoria a Corto Plazo (en adelante MCP). A continuación se presentan algunas investigaciones relacionadas con los procesos cognitivos implicados en la lectura de los estudiantes sordos, como son las RCGF y la MCP, con la finalidad de acercarnos a comprender las características reales de las dificultades lectoras presentadas por los lectores sordos.

Existen varios modelos que pretenden explicar el rol que cumple la información proveniente de la relación entre ortografía y fonología en la lectura. El modelo de acceso directo propone que los lectores acceden al léxico interno utilizando una ruta semántica, plantea que la extracción de rasgos y el reconocimiento de las letras tienen lugar gracias al procesamiento en paralelo (simultáneo). El modelo propone que los lectores emplean los conocimientos implícitos de la redundancia ortográfica para facilitar la percepción de las letras que no han sido procesadas por completo. Este proceso de reconocimiento primario constituye el input para un proceso de reconocimiento secundario que permite el acceso directo a los significados de las palabras. Foster y Chambers (1973) postulan que las palabras se reconocen, en primer lugar, de forma visual y por ello tendemos a identificar las palabras reales de un modo más rápido que las seudopalabras.

Por otra parte, el modelo de acceso indirecto propone que los lectores acceden al léxico interno utilizando una ruta fonológica, postula que los lectores generan el código fonológico para una palabra basándose en el conocimiento de las correspondencias entre grafemas y fonemas y este código es usado para acceder al léxico mental. Este modelo sugiere que la extracción de rasgos y el reconocimiento de letras ocurren gracias al procesamiento en serie (letra a letra) y que la identificación de palabras tiene lugar a través de una codificación fonética por turno de cada letra, utilizando las RCGF para acceder a los nombres de las palabras y sus significados. Un tercer modelo plantea que ambas rutas (fonológica y semántica) son usadas en el deletreo, ya que el deletreo de una palabra puede ser derivado de la aplicación de las RCGF o puede simplemente ser leído desde su correspondiente entrada en el léxico.

En este sentido, Carr (1981) plantea que un factor relevante en las dificultades lectoras es el déficit en la codificación fonológica y semántica. La habilidad para analizar los sonidos del habla y hacer conexiones entre las categorías abstractas del sonido y los patrones ortográficos se denomina conciencia fonológica. A partir de ello, se asume que los malos lectores carecen de conocimientos fonológicos suficientes para usar las reglas fonéticas, es decir, para trasladar lo impreso a la palabra hablada y viceversa.

\section{REGLAS DE CORRESPONDENCIA GRAFEMA-FONEMA}

De acuerdo con los modelos de adquisición de la ortografía (Wolf, Vellutino y Berko 1999) el conocimiento de las relaciones entre grafemas y fonemas juega dos funciones 
fundamentales en el desarrollo del deletreo. Primero, el conocimiento de las RCGF proporciona un mecanismo de apoyo al deletreo de palabras. Segundo, el conocimiento de las RCGF funciona como una herramienta mnemónica, permitiendo a los aprendices retener la información específica de las letras sobre las palabras individuales en la memoria. Igualmente, el uso de la información grafema-fonema se relaciona con la familiaridad o dificultad de las palabras al leer y con las habilidades de los lectores.

En una investigación realizada por Waters y Doehring (1990), señalan que el conocimiento de las RCGF es relevante para leer palabras nuevas y seudopalabras, ya que al no existir una entrada léxica para ellas no se conoce su pronunciación. Las investigaciones muestran que en el transcurso del aprendizaje de la lectura, los niños oyentes extraen información de la relación entre grafías y fonos del lenguaje y que el uso y conocimiento de esta información se relaciona sistemáticamente con la habilidad lectora. Los niños que aprenden las RCGF tienen una poderosa herramienta para decodificar las palabras que no están en su vocabulario visual en un código fonológico conocido. Por otra parte, distintas investigaciones plantean que los niños oyentes tienen algún nivel de conocimiento explícito sobre la conciencia fonológica que facilita el éxito en el inicio de la lectura. La conciencia fonológica implica el acceso consciente al nivel fonológico del habla y la manipulación cognitiva de las representaciones fonológicas. Los lectores instruidos en conciencia fonológica se desempeñan mejor en tareas de lectura, de reconocimiento de palabras y de deletreo. En definitiva, el conocimiento fonológico contribuye al éxito de la lectura.

Con el objeto de explorar la incidencia de las RCGF en buenos y malos lectores, Waters, Bruck y Seidenberg (1985) estudiaron una muestra de 150 estudiantes oyentes de $3^{\text {er }}$ grado y los dividieron en grupos de buenos y malos lectores y deletreadores. Los resultados del estudio indican que los niños de $3^{\text {er }}$ grado usan procesos similares para leer y deletrear, utilizando en ambas tareas las RCGF. Los autores señalan que el uso de RCGF es un factor diferenciador entre buenos y malos lectores. En otras palabras, el uso de las RCGF está a la base de las buenas habilidades en lectura y deletreo. Asimismo, aunque los malos lectores y los malos deletreadores tienen un conocimiento menor de las RCGF, intentan aprovechar este tipo de información. Este estudio señala que los niños de $3^{\text {er }}$ grado usan las RCGF tanto en las tareas de lectura como de deletreo. Si bien todos los intentan usar el principio de correspondencia entre grafemas y fonemas, los niños que poseen buenas habilidades en lectura y deletreo tienen mayor conocimiento de este principio. Los resultados del estudio muestran que los malos deletreadores comparten un déficit común en el uso y conocimiento del principio grafema-fonema en la lectura y el deletreo, independiente de sus habilidades en comprensión lectora.

En un estudio realizado para conocer el rol de la información lingüística de base auditiva y visual en tareas de deletreo, Waters, Bruck y Malus-Albramovitz (1988) estudiaron una muestra de 158 estudiantes oyentes entre $3^{\circ}$ y $6^{\circ}$ grado, divididos en grupos de buenos y malos lectores. Sus resultados mostraron que los estudiantes no deletreaban todas las palabras con igual facilidad, presentaban menos dificultades en las palabras con menor cantidad de segmentos ambiguos y en las que podían ser derivadas de las RCGF. Las autoras concluyen que los estudiantes de educación primaria muestran conocimientos de las RCGF, de las reglas que gobiernan las convenciones ortográficas del lenguaje y de la manera en que la ortografía representa significados. Señalan que a partir de $3^{\text {er }}$ grado los estudiantes intentan usar todos los conocimientos del deletreo. No obstante, 
los estudiantes con malas habilidades de deletreo poseen un menor conocimiento fonológico, ortográfico, morfológico y visual. Concluyen que los malos deletreadores usan los mismos procesos que los buenos deletreadores, pero dependen más de la información visual. Los investigadores que han estudiado el rol de la información grafema-fonema en las habilidades de deletreo en los niños oyentes concluyen que ambos recursos (visuales y fonológicos) son necesarios para el desarrollo normal de la lectura.

En este sentido, Boder (1973 citado por Dood 1980) identificó un grupo de estudiantes con dificultades en lectura y/o deletreo, diagnosticados con dislexia fonológica. Estos estudiantes eran incapaces de analizar fonéticamente las palabras, leían mal palabras nuevas y seudopalabras, al deletrear omitían sílabas y añadían letras. Un segundo grupo analizado presentaba dislexia visual, estos estudiantes podían analizar seudopalabras, pero tenían muy poco vocabulario visual. Respecto a este estudio, Dood (1980) comenta que si tanto la información visual como la fonológica son necesarias para el desarrollo normal de la lectura y el deletreo, cabe la hipótesis de que los niños sordos al carecer de información fonológica confíen exclusivamente en la información visual. De ser así, deberían desempeñarse como los disléxicos fonológicos. Sin embargo, existe poca evidencia de tal semejanza, ya que si bien es cierto un grupo importante de personas sordas presenta retraso en la habilidad lectora, muchos de ellos desarrollan niveles aceptables de lectura.

Tomando como antecedentes este y otros estudios, Dood (1980) plantea que los sordos podrían usan un código fonológico que les permita desarrollar un sistema fonológico. Señala que la capacidad de codificar en forma fonológica también podría ser utilizada por estos lectores en las tareas de deletreo. En torno a esta hipótesis, Dood (1980) realizó una investigación con dos grupos; el primero conformado por 10 estudiantes oyentes con una edad promedio de 14 años y el segundo por 10 estudiantes sordos con una pérdida auditiva promedio de 99 decibelios, educados en programas orales y con el mismo promedio de edad. Una de las tareas consistía en deletrear palabras familiares a partir de la lectura labial. El patrón de errores mostró que tanto los sordos como los oyentes distinguen mejor las palabras que tienen una correspondencia directa entre fonema y grafema. También se observó un grupo de niños sordos que podía extraer información fonológica desde un input visual único, en este caso la lectura labial. La autora descubrió una interacción entre la regularidad ortográfica y el estatus de audición. Los niños oyentes cometían menos errores cuando deletreaban palabras de ortografía regular $(12,2 \%)$ que cuando deletreaban palabras de ortografía irregular $(37,7 \%)$. En cambio, los niños sordos cometían prácticamente el mismo número de errores con palabras de ortografía regular $(28,8 \%)$ que con palabras de ortografía irregular $(27,2 \%)$.

Dood (1980) concluye que los niños sordos pueden usar códigos fonológicos cuando la información gráfica inequívoca no está disponible. Señala que no es sorprendente que los sordos presenten habilidades de deletreo, ya que han demostrado ser capaces de usar tanto códigos fonológicos como códigos visuales, aunque probablemente este último sea el preferido en la mayoría de las tareas. Estos planteamientos comienzan a proyectar luz sobre investigaciones posteriores, que postulan que el desarrollo de un código fonológico no es exclusivamente auditivo, sino que también puede ser derivado de la visualidad (lectura labial, dactilología, ortografía, palabra complementada, etc.).

Aunque es posible que los sordos que se comunican en forma oral usen la información proveniente de la relación grafema-fonema para decodificar palabras, parece menos 
probable que la información fonológica de base auditiva juegue un rol importante en el reconocimiento de palabras en los sordos que utilizan lenguajes de signos. Respecto de esta controversia, existen diferentes hipótesis sobre cómo los individuos sordos que signan, pero no usan códigos fonológicos, reconocen palabras escritas, ya que se ha comprobado que no existe relación entre signos y ortografía. Un primer antecedente lo encontramos en Locke y Locke (1971), quienes explican que los sordos carentes de lenguaje oral inteligible usan la dactilología (alfabeto manual) cuando se les pide recordar una serie de letras impresas. Hanson, Liberman y Shankweiler (1984) confirmaron y extendieron estos hallazgos a niños sordos de 6 a 11 años que participaban en tareas de recuerdo serial. Los estímulos presentados fueron una serie de letras con semejanza visual, dactílica y fonológica, junto con una serie control. Los resultados advierten que la dactilología es usada por las personas sordas como un sistema de codificación permanente, ya que la codificación dactílica se aprecia en el estudio como un código lingüístico relevante para los lectores sordos. Estos resultados también han sido corroborados en estudiantes sordos chilenos que usan Lengua de Signos Chilena, LSCh (Herrera 2003). Los estudios antes mencionados evidencian que los estudiantes sordos considerados buenos lectores usan más la dactilología que los estudiantes sordos considerados lectores deficientes.

Al respecto, Treiman y Hirsh-Pasek (1983) sugieren que el vínculo necesario para establecer relaciones entre lengua de signos y ortografía en los niños sordos podría ser la dactilología. Las autoras creen que así como los niños oyentes son capaces de decodificar palabras impresas no familiares a partir del significado de una forma hablada, los niños sordos pueden ser capaces de decodificar palabras impresas a partir de la dactilología de palabras familiares que tengan significado en su lenguaje.

Hirsh-Pasek (1982 citado por Hirsh-Pasek 1987) comprobó que los lectores sordos novatos de $3^{\text {er }}$ grado distinguen claramente signos desde la dactilología. Señala que así como los niños oyentes segmentan palabras habladas en fonemas, los niños sordos que signan segmentan palabras dactilografiadas en queiremas ${ }^{1}$. De igual modo, Leybaert (2000) sugiere que la dactilología puede ayudar a los sordos signantes nativos a comprender que las palabras impresas están hechas de segmentos y desarrollar conocimiento de las RCGF. Igualmente, Puente, Alvarado y Herrera (2006) encontraron una fuerte correlación entre la habilidad de los estudiantes sordos para segmentar palabras en letras y la habilidad para segmentar palabras en dactilología, sugiriendo que los niños sordos utilizan la similitud entre dactilología y ortografía para establecer reglas de correspondencia entre queiremas y grafemas.

Maxwell (1984) observó que los estudiantes sordos identificaban con mayor claridad las palabras escritas que no conocían o que no tenían un equivalente en su lengua de signos (como las palabras función), cuando usaban la dactilología para recordarlas. Estas investigaciones dan cuenta de que la dactilología tiene el potencial de ayudar a los lectores sordos a descifrar palabras, sin embargo esta estrategia debe ser sistematizada, ya que los niños sordos no vinculan de forma espontánea la ortografía y la dactilología.

Padden (1993) realiza un estudio con una muestra de 40 niños sordos entre 4 y 10 años, con el objetivo de determinar la relevancia de la ortografía en la lectura. La autora analiza el desempeño en tareas de deletreo, según la posición inicial, media o final de las letras. Concluye que los niños sordos poseen y adquieren conocimientos de las RCGF

1 Forma o configuración que adopta la mano al realizar un signo o representar una letra del alfabeto latino. 
desplegadas en la ortografía de la lengua y son capaces de utilizarlas en el reconocimiento de estructuras ortográficas propias o ajenas a su lengua. En esta línea Padden y Ramsey (1993) señalan que cuando los niños sordos deletrean no vinculan palabras habladas con palabras impresas sino letras del alfabeto manual con palabras impresas. En palabras de las autoras, los sordos que emplean el lenguaje de signos crean vínculos desde su propio sistema, generando nuevas formas para desarrollar la competencia ortográfica.

Padden y Ramsey (1998) realizan un nuevo estudio con 135 estudiantes sordos e hipoacúsicos para analizar la comprensión en dactilología. Advierten que el uso de la dactilología correlaciona significativamente con las habilidades en Lenguaje de Signos Americano (en adelante ASL) y en lectura. Postulan que los estudiantes con mayor exposición al lenguaje de signos y la dactilología han construido un vínculo entre el inglés y otros indicadores que puede ser útil para considerar las habilidades en dactilología, separadamente de las habilidades para signar en ASL.

Alvarado, Puente y Herrera (2008) estudiaron el uso de códigos fonológicos y visuales en tareas de MCP y su relación con el desempeño en tareas de lectura y ortografía. Analizaron una muestra de 33 niños chilenos entre 7 y 16 años divididos en dos grupos; un grupo experimental conformado por 28 niños sordos y un grupo control conformado por 15 niños oyentes. Los autores concluyen que existe una correlación entre el uso de códigos dactílicos y ortográficos en los estudiantes sordos. Señalan que así como el conocimiento de las relaciones entre fonemas y grafemas facilita la lectura en los niños oyentes, el conocimiento de la relación entre queiremas y grafemas tiene el potencial de jugar un rol similar en los lectores sordos.

Por otra parte, Asensio, González y Carretero (1989) realizaron una investigación con el objeto de determinar la relación entre uso de Lengua de Signos Española (en adelante LSE) y el desempeño lector de estudiantes sordos. Seleccionaron una muestra de estudiantes sordos y oyentes escolarizados entre $1^{\circ}$ y $8^{\circ}$ grado de educación primaria, controlando que el método de aprendizaje de la lectura fuera alfabético, es decir, que empleara las RCGF. Los autores concluyen que los niños oyentes alcanzan el dominio del sistema de RCGF prácticamente en un año de instrucción lectora. Por el contrario, los niños sordos no alcanzan su dominio ni siquiera en el último año de instrucción de enseñanza primaria. Agregan que los niños sordos progresan sólo 0,1 años por cada año de progreso de sus compañeros oyentes. Los datos presentados por los autores muestran que los niños sordos alcanzan un dominio de las RCGF en $8^{\circ}$ grado de enseñanza primaria de un $73 \%$, porcentaje que no permite la automatización del sistema. Al respecto, Asensio, et al. (1989) indican que "esta falta de automatización del sistema obliga a los estudiantes sordos a continuar prestando atención consciente a este nivel de microprocesos lectores, cargando sobremanera su memoria operativa. De este modo, no puede liberar recursos atencionales y operativos para los macroprocesos lectores de carácter comprensivo" (p. 22).

Asensio et al. (1989) concluyen que los lectores sordos, al igual que los malos lectores, presentan serios problemas en la utilización de las RCGF y una deficiente utilización de las habilidades de segmentación. Agregan que los niños que tienen un buen dominio de la LSE presentan buenos resultados en las pruebas de lectura, tanto en la utilización de las RCGF a nivel léxico como en las operaciones de segmentación subléxica. Sin embargo, no ocurre lo mismo con los niños sordos que no dominan correctamente la LSE. Concluyen que el dominio de las RCGF y las habilidades de segmentación son fundamentales para el 
proceso de aprendizaje de la lectura de los estudiantes sordos, ya que ambos mecanismos permiten el establecimiento de una estrategia alfabética de lectura.

Para Schaper y Reitsma (1993), el dominio de las RCGF promueve la adquisición de las habilidades lectoras al menos en tres formas. Primero, el uso de las RCGF en la palabra impresa permite al lector novato decodificar palabras desconocidas. Segundo, la valoración de una estrategia fonológica en el aprendizaje de la lectura facilita la adquisición del conocimiento de palabras impresas, ya que ésta atrae la atención a la información sobre las letras que componen una palabra más que a sus características gráficas. Una estrategia fonológica puede funcionar como un mecanismo para adquirir habilidades en la identificación rápida y exacta de palabras por medio de una estrategia visual "directa", que es una condición muy importante en la lectura eficiente de un lector. Y tercero, en términos de comprensión del texto, la codificación fonológica de palabras impresas permite al lector codificar lo impreso en una forma más durable de almacenamiento en la MCP, y por ende retener la información para interpretar oraciones más largas.

\section{MEMORIA A CORTO PLAZO}

Entre los procesos cognitivos implicados en el aprendizaje de la lectura y en cualquier otro aprendizaje, la memoria es primordial. Como sabemos la memoria junto con la atención y la percepción forman el núcleo de los procesos psicológicos básicos. En concreto, el desarrollo de la memoria tendrá gran influencia en el aprendizaje y en el uso del lenguaje. Por ello, el estudio de la memoria ha estado ligado estrechamente al uso de material lingüístico. La capacidad de memoria ha sido estudiada a través de la presentación de materiales lingüísticos diversos como palabras que riman, sinónimos, sílabas sin sentido, listas de palabras, secuencias de letras, etc. Igualmente se ha estudiado el tiempo que el material lingüístico está presente en cada una de las memorias (sensorial, MCP y memoria a largo plazo); según sea el material utilizado, aparece sistemáticamente una serie de efectos. Debido al interés en el estudio de la memoria en la población sorda, son destacables el efecto rima y el efecto longitud de las palabras.

En el efecto rima los estímulos semejantes tienden a confundirse obligando al sistema de procesamiento a esforzarse más para diferenciarlos. Una lista de palabras que rimen será más difícil de recordar que una lista de palabras que no rimen, si para el repaso se utiliza el componente fonológico. En los oyentes este efecto se produce con alta frecuencia, es decir, recuerdan menos palabras cuando éstas riman entre sí. En cambio, en los sordos este efecto no se produce ya que tienden a recordar de igual forma palabras que riman y palabras que no. Para Torres (1998), este hecho muestra que los sordos no utilizan la fonología al mismo nivel que los oyentes.

En el efecto longitud de las palabras los oyentes tienden a recordar mejor las listas de palabras bisílabas que las listas de palabras polisílabas. En cambio, los sordos tienden a recordar el mismo número de palabras independientemente de la longitud de ellas. Para Torres (1998) esta diferencia en los resultados entre sordos y oyentes se atribuye al distinto conocimiento y uso que unos y otros hacen de los códigos fonológicos. Asimismo, las características del material verbal influyen en los rendimientos de la memoria.

El rol que juega en el reconocimiento de palabras la información fonológica ha sido considerado un factor importante en la función de la memoria durante la lectura para los 
oyentes, puesto que la lectura involucra múltiples habilidades (reconocimiento de palabras, procesamiento sintáctico, inferencias, etc.) y todas ocurren en paralelo, la información debe ser temporalmente retenida en alguna especie de memoria de trabajo o MCP. Existe evidencia considerable de que la información verbal es retenida en la MCP en códigos basados en el habla o códigos fonológicos, independientemente de si el material para recordar es presentado auditiva o visualmente (Conrad 1979).

Del mismo modo, muchos teóricos han sugerido que la MCP juega un rol crítico en la comprensión lectora. Su mayor influencia puede estar en el procesamiento oracional e intraoracional, ya que tanto el almacenamiento como el procesamiento de la información ocurren en la MCP. Actualmente existe consenso respecto a que el uso de un código fonológico en la MCP es crítico para llevar a cabo un procesamiento eficiente, especialmente para un lenguaje fonético, temporal y secuencial como el lenguaje oral (Paul 1998). Ahora bien, aunque se han usado pruebas indirectas para explorar el uso de códigos fonológicos en la lectura de los sordos, existen numerosas investigaciones de su capacidad de MCP, del uso de diferentes tipos de códigos de memoria en las tareas de MCP y de la relación entre estas variables y los logros en las pruebas de lectura. En general se ha descubierto una capacidad de memoria menor en los individuos sordos que en los oyentes, lo que se atribuye a un uso menos eficiente de los códigos fonológicos en la MCP o a que la población sorda usa códigos de memoria cualitativamente distintos a los utilizados por la población oyente (Waters y Doehring 1990).

Para Conrad (1979), la MCP se basa en algún tipo de código acústico relacionado con el habla. Este investigador estudió los errores que cometen los sujetos cuando reproducen una lista de letras (habladas o por escrito) que han visto durante medio segundo en la pantalla de un computador. Los errores de los oyentes se relacionan más con los sonidos de las letras que con las formas de las letras. Según el autor, los oyentes vemos grafemas pero almacenamos fonemas. Respecto de los sordos, determina que existen sordos que emplean códigos de naturaleza acústico-articulatoria y otros que utilizan algún tipo de código de mediación que podría depender de los signos. En este sentido, Craine (1977 citado por Torres 1998) sostiene que los códigos de naturaleza acústico-articulatoria son de primordial importancia para los oyentes y los usan casi de forma exclusiva, en cambio los sordos utilizan este código en los procesos de la MCP sólo si su lenguaje oral es lo suficientemente fluido, de no ser así, se inclinan a utilizar un código visual.

Por otra parte, los resultados respecto del uso de códigos fonológicos en los sordos que utilizan la lengua de signos para comunicarse son inconsistentes. Locke y Locke (1971) compararon dos grupos de 28 sujetos sordos entre 14 y 20 años, divididos en sujetos con habla inteligible y sujetos con habla ininteligible, además de un grupo control de 26 sujetos oyentes entre 10 y 14 años. Ello con el objetivo de analizar los errores cometidos en una tarea de recuerdo y reconocimiento de distintas series de letras. En un análisis posterior de los errores, se confirmó que algunos estudiantes sordos confían en el código dactílico (proveniente de la información visual y cinética de la configuración de la mano) como código de almacenamiento en la MCP. Se observó que los oyentes cometen un alto número de errores de tipo fonológico y que los sordos cometen errores de tipo visual. Los autores concluyen que existe una conexión notoria entre las capacidades de comunicación del sordo y las estrategias de codificación en la MCP.

En este sentido, Liben y Drury (1977) observaron que incluso los niños sordos más jóvenes (6 años) se valían del alfabeto manual para designar las letras en las tareas de 
MCP. Usando palabras del inglés como estímulos, Odon, Blanton y McIntyre (1970) mostraron que el recuerdo de las personas sordas mejora empleando palabras que tienen traducción al lenguaje de signos (en este caso, ASL). Igualmente, Moulton y Beasley (1975) probaron que los sordos recuerdan mejor aquellos pares de signos que tienen parámetros formativos similares que los pares de signos sin similitud formacional. Treiman y Hirsh-Pasek (1983) descubrieron que los signantes nativos cometían más errores en oraciones con similitud queirémica (dactílica) que en oraciones control. De este modo, la evidencia de códigos visuales, tanto dactílicos como signados, en la población sorda ha sido advertida desde hace años en distintos estudios.

Interesados por estudiar el uso de códigos de MCP en la población sorda, Wallace y Corballis (1973) realizan un estudio cuyo objetivo fue investigar el procesamiento en la MCP de secuencias de letras presentadas visualmente. Para ello examinan el desempeño de tres grupos; un grupo de 8 sujetos sordos con educación oral, un grupo de 8 sujetos sordos con educación basada en ASL y un grupo control de 8 sujetos oyentes. Los resultados de este estudio demuestran que los sordos que reciben educación oral utilizan indistintamente códigos fonológicos y dactílicos. En cambio, el grupo de sordos con educación basada en signos utiliza exclusivamente códigos dactílicos. Estos resultados proponen la existencia de componentes visuales y cinéticos en la MCP de los sordos. Sobre la base de estos resultados, los autores proponen que para los sordos educados con lengua de signos, la dactilología provee un código de almacenamiento alternativo eficaz.

Otra línea de investigación ha relacionado la habilidad sintáctica con el recuerdo serial de la información. Lake (1980) llevó a cabo una tarea de MCP para dígitos, dividiendo a los estudiantes sordos en dos grupos; un grupo que respondió consistentemente al orden serial de la información y otro grupo que no lo hizo. Además, analizó muestras de escritura de estos niños y en este análisis verifica que la habilidad para almacenar temporalmente información secuencial en la MCP está relacionada con las competencias sintácticas, ya que éstas proporcionan información sobre el orden de las palabras.

Por otra parte, Hanson (1982) comparando un grupo de adultos sordos con uno de adultos oyentes observa que los sordos, que emplean la lengua de signos aprendida de sus padres sordos, usan códigos fonológicos en las tareas de memoria y ello está relacionado con su capacidad de memoria. Asimismo, Lichtenstein (1982 citado por Waters y Doehring 1990) señala que los adultos sordos que no son signantes nativos, pero cuyo modo primario de comunicación es la lengua de signos, también emplean códigos fonológicos y que el uso de estos códigos está relacionado con la capacidad de MCP, pero no con la inteligibilidad del habla.

Igualmente, Hanson, Liberman y Shankweiler (1984) plantean que los niños sordos signantes nativos y buenos lectores emplean códigos fonológicos para los propósitos de memoria. Nuevamente se llega a la conclusión de que los sordos considerados buenos lectores utilizan tanto códigos fonológicos como códigos dactílicos a la hora de retener letras impresas en la MCP. Sin embargo, la mayoría de los estudios revelan que los sordos que utilizan lengua de signos, pero que no utilizan códigos fonológicos para los propósitos de MCP, utilizan una variedad de otros códigos de almacenamiento en la MCP (Lichtenstein 1998). Los sordos signantes pueden usar códigos de memoria basados en las características visuales de las letras (Locke y Locke 1971), en la dactilología a través de códigos dactílicos (Hanson et al. 1984) o en las características formacionales de los signos como la orientación y configuración de la mano, el lugar de articulación o el movimiento (Shand 1982). 
La mayor evidencia del uso de códigos manuales proviene de los hallazgos de un efecto de similitud queirémica o formacional en la memoria para los signos. Este efecto es similar al efecto de similitud fonológica para las palabras habladas en los oyentes. Al respecto, Klima y Bellugi (1980) y Hanson (1982) señalan que los sordos que signan tienen mejor memoria para listas de signos diferentes en sus parámetros formativos que para listas de signos con parámetros formativos similares. En este sentido, Bellugi, Klima y Siple (1975) señalan que los sordos recuerdan peor las listas de signos que se diferencian en un solo parámetro formativo. Esto es similar a los hallazgos con oyentes, quienes al recordar listas de palabras recuerdan peor las palabras que se diferencian en un solo fonema. Dichos resultados son consistentes con la teoría que propone que los signos son codificados por los sordos en términos de parámetros formativos simultáneos, independientes, específicos de la lengua de signos y esencialmente arbitrarios en términos de significado.

Desde esta perspectiva, Shand (1982) sostiene que los sordos profundos eficientes en lenguaje de signos decodifican los estímulos ortográficos en un código de base signada, sugiriendo que la preferencia de cierto sistema de codificación para el recuerdo del material lingüístico no está necesariamente limitada a un código fonológico. En su estudio expone que la codificación de los estímulos ortográficos, por parte de los sordos, en códigos de base queirológica, es más extraordinaria que la codificación de estos estímulos en códigos fonológicos por parte de los oyentes. Agrega que para los sordos que utilizan la lengua de signos como primera lengua, es más fácil almacenar el material lingüístico en códigos conocidos como los códigos de base visual. Dicha facilidad se debe a la tendencia a codificar el material lingüístico en un sistema basado en códigos lingüísticos primarios para el sujeto. Este sistema es fonológico para la mayoría de los oyentes, pero podría ser signado para aquellos sordos cuyo medio principal de comunicación es la lengua de signos. En su investigación Shand (1982) también declara que existe en los sordos congénitos una tendencia a codificar el estímulo lingüístico en términos de propiedades formativas, incluso cuando el estímulo no es un signo sino una palabra escrita. Esto es especialmente llamativo debido al hecho de que tal codificación involucra, por una parte, una codificación queirológica más que fonológica, y por otra, una traducción desde la palabra impresa a un código queirológico, incluso aunque la presentación y el recuerdo sean realizados a partir de palabras habladas. El autor concluye que el tipo de código usado por los sujetos se relaciona con su experiencia lingüística.

Igualmente Hamilton y Holzman (1989) señalan que el tipo de código de almacenamiento en la MCP está determinado por las características del estímulo. En tanto el tipo de estímulo afecta el uso de códigos en la MCP. En base a esta evidencia se deduce que los sordos que adquieren el lenguaje de signos como lenguaje primario emplean códigos de base signada para los procesos de MCP. En consecuencia, el código utilizado depende al mismo tiempo de la experiencia lingüística y del input del código.

En esta línea Mayberry y Waters (1991) investigaron el recuerdo de signos y dactilología en 43 niños sordos entre 7 y 15 años. Para ello usaron 105 signos del ASL y 91 palabras dactilografiadas. Las listas de signos se realizaron con signos compuestos por uno, dos o tres signos y las palabras dactilografiadas de palabras con tres, cuatro o cinco letras. Los resultados de este estudio sugieren que no todos los estímulos se recuerdan por igual, el recuerdo de signos es completamente diferente del recuerdo de palabras dactilografiadas. Las autoras plantean que la razón de estos resultados puede 
estar relacionada con el hecho de que la organización lexical de las palabras dactilografiadas y los signos es diferente. Aunque ambos estímulos son espaciales y la forma y orientación de la mano son importantes para la formación e identificación lexical, en los signos las formas de la mano coocurren con los movimientos, hecho crucial para la identificación y formación del signo. En cambio, en la dactilología las formas de la mano son secuenciadas una después de la otra para formar una palabra. En suma, las palabras dactilografiadas comparten con las habladas un mayor grado de secuencialidad que con los signos y he allí la importancia de incorporar la dactilología en la educación de los sordos con el fin de ampliar el reconocimiento fonológico y la capacidad de segmentar palabras (Herrera 2003).

Por otra parte, Mayberry y Waters (1991) descubren que la capacidad de almacenar signos en la memoria no incrementa después de los 10 o 12 años. En cambio la memoria para las palabras dactilografiadas continúa incrementando a los 13 o 15 años. Entre los 7 y 15 años la memoria de signos incrementa en un 50\%, mientras la memoria de palabras dactilografiadas incrementa en un $103 \%$. Las autoras explican que los signantes, al igual que los hablantes, usan códigos fonológicos para el recuerdo, incluso aunque la fonología sea gestual en vez de hablada. Los hablantes tienden a recordar mejor las palabras habladas que oyen y los signantes los signos que ven (Krakow y Hanson 1985). Estas similitudes sugieren que los procesos de memoria para las palabras y los signos son similares, aunque la percepción y los modos de producción no lo sean. La investigación acumulada muestra que la variación en el input del lenguaje de signos durante la infancia puede afectar el desarrollo del lenguaje de signos al nivel de memoria y producción de palabras, tanto para signos como para dactilología.

\section{DISCUSION Y CONCLUSIONES}

A la luz de las investigaciones revisadas es posible afirmar que el uso de RCGF es un factor diferenciador entre buenos y malos lectores (sean sordos u oyentes), ya que los niños con buen nivel lector y buenas habilidades de deletreo dominan mejor dichas correspondencias. Otro dato interesante es que tanto los lectores novatos como los malos lectores poseen conocimientos insuficientes de las RCGF, pero aún así intentan usar este tipo de información durante la lectura. También se puede indicar que ya que el deletreo supone competencias para segmentar correctamente los fonemas, los estudiantes con escasas habilidades de deletreo poseen menos conocimientos fonológicos, ortográficos, morfológicos y visuales que los lectores más hábiles en las tareas de deletreo.

Igualmente, se puede señalar que tanto los lectores principiantes, los malos lectores y, en nuestro caso, los lectores sordos son menos conscientes de las RCGF del lenguaje. Concretamente en los sordos esto puede deberse a la falta de información auditiva para desvelar estas correspondencias. Sin embargo, como se ha comprobado, es posible que los lectores sordos accedan a esta información a través de vías visuales complementarias como la lectura labial, la palabra complementada, la dactilología, la ortografía, etc., que les permita beneficiarse del conocimiento de las correspondencias grafema-fonema para lograr mejores niveles de reconocimiento de palabras y comprensión lectora. Finalmente, ya que el conocimiento de las RCGF es fundamental en un sistema de escritura alfabético como el nuestro y debido a que este conocimiento parece elemental en la etapa inicial 
del proceso lector, deberían posibilitarse todas las vías de acceso a la información a los lectores sordos para que puedan crear vínculos que les permitan representar la información grafema-fonema.

La investigación respecto de los procesos de MCP en las personas sordas genera tres conclusiones relevantes. Primero, en general, se ha probado con un variado tipo de material lingüístico que el espacio de memoria en los sordos es más limitado que en las personas oyentes. Estos resultados no sólo se aplican al material verbal, ya que distintos investigadores han señalado que la capacidad de los sordos para almacenar una secuencia de signos en la MCP no es tan buena como la retención que realizan los oyentes en una secuencia de palabras. Las diferencias en capacidad de MCP encontradas en los sordos se deben no solo al desconocimiento del material lingüístico, sino también a los procesos cognitivos involucrados en la codificación del material lingüístico. En segundo lugar, los procesos de MCP en los estudiantes sordos son diferentes a los de la población oyente, tanto en calidad como en cantidad. Tercero, existen diferencias individuales importantes entre la población sorda respecto a los tipos de códigos empleados en el almacenamiento de la información lingüística. En este sentido, se ha encontrado evidencia de que los sordos son capaces de usar códigos visuales, dactílicos, signados y hablados para retener información en la MCP.

Respecto a los procesos de MCP y las habilidades de lectura, la literatura reporta que los problemas relativos a la MCP y a los procesos de codificación han sido asociados con las dificultades de los niños sordos para aprender a leer y estos procesos son probablemente más críticos cuando aprenden un lenguaje que no conocen, especialmente al enfrentarse con su forma escrita. Segundo, existen investigaciones que muestran correlaciones positivas entre MCP y habilidades de lectura o escritura, en estos estudios se indica la relación entre almacenamiento de la información secuencial y habilidades sintácticas. Es decir, independientemente de cuál sea el lenguaje primario, los individuos que aprenden a leer y escribir deben desarrollar estrategias para almacenar información secuencial. En tercer lugar, diversos estudios han descubierto que las diferencias en la capacidad de MCP en los estudiantes sordos y las estrategias de codificación están relacionadas con el nivel general de logro en lectura. En cuarto lugar, tanto las consideraciones teóricas como los datos disponibles han conducido a algunos teóricos a suponer que las limitaciones en los procesos de codificación y la capacidad de MCP afectan el procesamiento de la sintaxis y la adquisición de la gramática de los estudiantes sordos.

Sabemos que la fonología juega un rol esencial en el almacenamiento de la información lingüística en los oyentes. Asimismo, se ha comprobado que para los sordos la fonología no es exclusivamente auditiva, sino que también puede contener componentes visuales y cinéticos. Los resultados de los trabajos en MCP con sordos indican que éstos utilizan múltiples códigos de almacenamiento de la información. Cuando las palabras son representadas por escrito, los sordos usan códigos ortográficos y semánticos. Es decir, se fijan tanto en la forma de la palabra escrita como en su contenido. Cuando se trata de signos, los sordos atienden a los parámetros formativos del signo y al contenido semántico.

Por lo tanto, el mejoramiento y enriquecimiento de las habilidades de MCP y RCGF en los sordos requieren incorporar e integrar en la didáctica de la lectoescritura la utilización de todas las modalidades sensoriales que faciliten la vinculación de la información gráfica proveniente del texto escrito, con códigos de procesamiento de la información basados en experiencias visuales, signadas, dactílicas y ortográficas que les permitan acceder a la información escrita. 


\section{BIBLIOGRAFIA}

Alvarado, J. M., A. Puente \& V. Herrera (2008). Visual and phonological coding in working memory and orthographic skills of deaf children using Chilean Sign Language. American Annals of the Deaf 152 (5): 299-310.

Asensio, M., J. L. González y M. Carretero (1989). Buenos y malos lectores sordos. Boletín de Ciencias de la Educación 15: 5-39. Madrid.

Bellugi, U., E. S. Klima \& P. Siple (1975). Remembering in signs. Cognition 3 (2): 93-125.

Carr, T. H. (1981). Building theories of reading ability: on the relation between individual differences in cognitive skills and reading comprehension. Cognition 9 (1): 73-114.

Conrad, R. (1979). The Deaf School Child. London: Harper \& Row.

Dood, B. (1980). The spelling abilities of profoundly pre-lingually deaf children. En U. Frith (ed.) Cognitive Processes in Spelling. London: Academic Press.

Foster, K. I. \& S. M. Chambers (1973). Lexical access and naming time. Journal of Verbal Learning and Verbal Behavior 12: 627-635.

Hanson, V. (1982). Short-term recall by deaf signers of American Sign Language: Implication of encoding strategy for order recall. Journal of Experimental Psychology: Learning. Memory and Cognition 8 (6): 572-583.

Hanson, V., I. Liberman \& D. Shankweiler (1984). Linguistic coding by deaf children in relation to beginning reading success. Journal of Experimental Child Psychology 37 (1): 378-393.

Hamilton, H. \& T. G. Holzman (1989). Linguistic encoding in short-term memory as a function of stimulus type. Memory \& Cognition 17 (5): 541-550.

Herrera, V. (2003). Desarrollo de habilidades lectoras en sujetos sordos signantes, a partir del uso de códigos dactílicos. Tesis doctoral no publicada. Universidad Complutense de Madrid.

Hirsh-Pasek, K. (1987). The metalinguistic of fingerspelling: An alternate way to increase reading vocabulary in congenitally deaf readers. Reading research Quarterly XXII (4): 455-473.

Krakow, R. \& V. Hanson (1985). Deaf signers and serial recall in the visual modality: Memory for signs, fingerspelling and print. Memory \& Cognition 13 (3): 265-272.

Klima, E. \& U. Bellugi (1980). The Sign of Language. Cambridge. Harvard University Press.

Lake, D. (1980). Syntax and sequential memory in hearing impaired children. En. H. N. Reynolds \& C. M. Williams (eds.). Proceeding of Gallaudet conference on reading in relations to deafness. Washington, D.C.: Gallaudet college.

Leybaert, J. (2000). Phonology acquired through the eyes and spelling in deaf children. Journal of Experimental Child Psychology 75: 291-318.

Liben, L. \& A. Drury (1977). Short-term memory in deaf and hearing children in relation to stimulus characteristics. Journal of Experimental Child Psychology 24: 60-73.

Lichtenstein, E. H. (1998). The relationships between reading processes and English skills of deaf college students. Journal of Deaf Studies and Deaf Education 3 (2): 80-134.

Locke, J. \& V. Locke (1971). Deaf childrenís phonetic, visual, and dactylic coding in grapheme recall task. Journal of Experimental Psychology 89 (1): 142-146.

Mayberry, R. \& G. Waters (1991). Children's memory for sign and fingerspelling in relation to production rate and sign language input. En P. Siple \& S. Fischer (eds.). Theoretical Issues in Sign Language Research. Volume 2: Psychology. Chicago: The University of Chicago Press.

Maxwell, M. (1984). A deaf child's natural development of literacy. Sign Language Studies 44: 191-224.

Moulton, R. \& D. Beasley (1975). Verbal coding strategies used by hearing-impaired individuals. Journal of Speech and Hearing Research 18: 559-570.

Odon, Blanton y McIntyre (1970). Coding medium and word recall by deaf and hearing subjects. Journal of Speech and Hearing Research 13: 54-58.

Paul, P. V. (1998). Literacy and Deafness: the development of reading, writing and literate thought. Boston: Allen and Bacon. 
Padden, C. (1993). Lessons to be learned from the young deaf orthographer. Linguistics and Education 5: 71-86.

Padden, C. \& C. Ramsey (1993). Deaf culture and Literacy. American Annals of the Deaf 138 (2): 96-99.

Padden, C. \& C. Ramsey (1998). Reading ability in signing deaf children. Topics in Language Disorders 18 (4): 30-46.

Puente, A., J. Alvarado \& V. Herrera (2006). Fingerspelling and sign language as alternative codes for reading and writing words for Chilean deaf signers. American Annals of the Deaf. Vol. 151, N 3: 299-310.

Schaper, W. M. \& P. Reitsma (1993). The use of speech-based recoding in reading by prelingually deaf children. American Annals of the Deaf 138 (1): 46-54.

Shand, M. (1982). Sign-based short-term coding of American Sign Language signs and printed english words by congenitally deaf signers. Cognitive Psychology 14: 1-12.

Torres, S. (1998). Memoria, fonología y sordera. Rev. Fiapas 62: 25-28.

Treiman, R. \& K. Hirsh-Pasek (1983). Silent reading: insights from second-generation deaf readers. Cognitive Psychology 15: 39-65.

Wallace, G. \& M. C. Corballis (1973). Short-term memory and coding strategies in the deaf. Journal of Experimental Psychology 99 (3): 334-348.

Waters, G., M. Bruck \& M. Seidenberg (1985). Do children use similar processes to read and spell words? Journal of Experimental child Psychology 39 (3): 511-530.

Waters, G. \& D. Doehring (1990). Reading acquisition in congenitally deaf children who communicate orally: insights from an analysis of component reading, language and memory skills. En T. Carr \& B. Levy (eds.). Reading and its Development. Component skills approach. San Diego: Academic Press.

Wolf, M., F. Vellutino y J. Berko (1999). Una explicación psicolingüística de la lectura. En J. Berko \& N. Bernstein, Psicolingüística. Madrid: McGraw-Hill. 